\title{
Osteogénesis imperfecta: hallazgos clínicos y epidemiológicos en una serie de pacientes pediátricos
}

\author{
Francisco Cammarata-Scalisi ${ }^{1 *}$, Carlos Ramos-Urrea ${ }^{2}$ y Gloria Da Silva ${ }^{1}$ \\ ${ }^{1}$ Unidad de Genética Médica, Departamento de Puericultura y Pediatría, Universidad de Los Andes, Mérida, Venezuela; ${ }^{2}$ Departamento de Fisiología \\ de la Nutrición, Universidad Federal de São Paulo, São Paulo, Brasil
}

\begin{abstract}
Resumen
Introducción: La osteogénesis imperfecta (OI) es el trastorno óseo hereditario más común, con una incidencia de 1 en 10,000 a 25,000 nacimientos. Este trastorno está causado principalmente por mutaciones de los genes que codifican las cadenas del colágeno tipo I. En la mayoría de los casos, se presenta un patrón de herencia autosómico dominante. La OI se caracteriza principalmente por un aumento en la fragilidad ósea que da lugar a fracturas frecuentes que producen dolor, deformidad y discapacidad asociada con otras alteraciones. El objetivo del estudio fue exponer las características clínicas y epidemiológicas de una serie de pacientes pediátricos con diagnóstico de Ol evaluados en la Universidad de Los Andes. Métodos: El presente trabajo consiste en el análisis de una serie de 37 casos pediátricos con diagnóstico de Ol, de acuerdo a la clasificación clínica y radiológica de Sillence, evaluados en la consulta de la Unidad de Genética Médica de la Universidad de Los Andes, entre enero de 2006 y diciembre de 2018. Resultados: La Ol tipo I fue la de presentación más frecuente, con 31 pacientes (83.78\%). El fémur fue el hueso más afectado de manera conjunta. Las escleras azules fueron el hallazgo adicional más frecuente, en 32 pacientes (86.49\%). Conclusiones: La Ol representa el principal motivo de consulta por alteraciones en el sistema esquelético en la Unidad de Genética Médica de la Universidad de Los Andes. Ante la amplia forma clínica de presentación, la evaluación debe ser individual e interdisciplinaria. A través de un estudio más profundo se podrá brindar el oportuno asesoramiento genético familiar.
\end{abstract}

Palabras clave: Osteogénesis imperfecta. Colágeno tipo I. Estudio clínico. Epidemiología. Clasificación de Sillence.

\section{Osteogenesis imperfect: clinical and epidemiological findings in a series of pediatric patients}

\begin{abstract}
Background: Osteogenesis imperfecta $(\mathrm{Ol})$ is the most common hereditary bone disorder with an incidence of one in 10,000-25,000 births. It is caused mainly by mutations in the genes that code for Type I collagen chains. In most cases, it shows an autosomal dominant inheritance pattern. Ol is characterized by an increase in bone fragility that leads to frequent
\end{abstract}

*Francisco Cammarata-Scalisi

E-mail: francocammarata19@gmail.com
Fecha de recepción: 17-03-2019

Fecha de aceptación: 06-06-2019 DOI: 10.24875/BMHIM. 19000030
Disponible en internet: 07-10-2019 Bol Med Hosp Infant Mex. 2019;76:259-264 www.bmhim.com 
fractures, which cause pain, deformity and disability associated with other alterations. The objective of this study was to present the clinical and epidemiological characteristics of a series of pediatric patients diagnosed with $\mathrm{Ol}$ evaluated at the University of Los Andes. Methods: A series of 37 pediatric cases with diagnosis of Ol according to the clinical and radiological classification of sillence is analyzed, which were evaluated in the medical genetics unit of the University of Los Andes consultation between January 2006 and December 2018. Results: Type I was the most frequent Ol type, with 31 patients (83.78\%). Additionally, the femur was the most affected bone. Blue scleras were the most frequent additional finding in 32 patients (86.49\%). Conclusions: Ol represents the main reason for consultation of alterations in the skeletal system in the medical genetics unit of the University of Los Andes. Given the broad clinical presentation, the evaluation must be individual and interdisciplinary. Further study will provide timely family genetic counseling.

Key words: Osteogenesis imperfecta. Type I collagen. Clinical study. Epidemiology. Sillence classification.

\section{Introducción}

La osteogénesis imperfecta $(\mathrm{OI})$ es el trastorno óseo hereditario más común ${ }^{1}$, con una incidencia de 1 en 10,000 a 25,000 nacimientos a escala mundial2,3. Este trastorno está causado principalmente por mutaciones de los genes que codifican a las cadenas del colágeno tipo I, y en la mayoría de los casos presentan un patrón de herencia autosómico dominante ${ }^{2}$.

Se han propuesto varios sistemas de clasificación para describir su variabilidad ${ }^{4}$. En 1979, Sillence, et al. propusieron una clasificación en cuatro tipos (I-IV) basada en los hallazgos clínicos y radiológicos de los pacientes ${ }^{5,6}$. Sin embargo, ante la extensa heterogeneidad genética, esta clasificación fue complementada por Rauch, et al., quienes la ampliaron a 16 tipos diferentes ${ }^{1,7}$, divididos en cinco grupos, basándose en la vía metabólica afectada con respecto a la síntesis, estructura, procesamiento, modificación postraduccional, plegamiento ${ }^{7}$, reticulación ${ }^{2,7}$, hidroxilación ${ }^{2}$, mineralización y diferenciación de colágeno tipo $\mathrm{I}^{2,7}$.

$\mathrm{La} O \mathrm{O}$ se caracteriza principalmente por el aumento en la fragilidad ósea ${ }^{1,3,7}$ y la disminución de la masa ósea ${ }^{4}$, lo que da lugar a fracturas frecuentes que producen dolor, deformidad y discapacidad'. Se puede asociar con huesos wormianos, hipoacusia, escleras azules, dentinogénesis imperfecta ${ }^{8-10}$, escoliosis, deformidad ${ }^{8}$, hiperlaxitud en la piel $^{9}$, hipermovilidad articular ${ }^{8,9}$, predisposición a presentar hematomas y talla baja ${ }^{8,10}$. Los tipos de Ol encontrados con mayor frecuencia en el entorno ortopédico pediátrico son los tipos I (leve fragilidad ósea), III (grave) y IV (moderada)'.

El diagnóstico de Ol puede ser sencillo cuando existen antecedentes familiares y las características típicas; sin embargo, puede dificultarse en pacientes con fenotipo leve o cuando se identifican hijos afectados con historial de fracturas 4 . El objetivo del presente estudio fue exponer las características clínicas y epidemiológicas de una serie de pacientes pediátricos con diagnóstico de Ol evaluados en la Unidad de Genética Médica de la Universidad de Los Andes (UGM-ULA).

\section{Métodos}

El presente trabajo consiste en una serie de 37 casos en los que se analizaron los datos clínicos y epidemiológicos disponibles de los pacientes evaluados en la consulta de la UGM-ULA de enero de 2006 a diciembre de 2018. Esta institución representa el único centro de referencia en genética médica del estado de Mérida. Se encuentra ubicado en el Instituto Autónomo Hospital Universitario de Los Andes, el cual se califica dentro del sistema de salud venezolano como un centro tipo IV. Además, sirve a los estados vecinos del suroccidente de Venezuela que no cuentan con servicios de genética.

Se incluyó solamente un paciente de cada familia, con diagnóstico clínico de Ol y edad comprendida entre 0 y 15 años, de acuerdo con la clasificación clínica y radiológica de Sillence. Estos pacientes presentaron un historial recurrente de más de dos fracturas y otros signos asociados con OI que orientaron su diagnóstico clínico. Los pacientes con diagnósticos de lesión no accidental fueron excluidos, así como los casos de osteoporosis idiopática o secundaria y displasias esqueléticas que pudieran predisponer a fracturas.

\section{Resultados}

Del total de pacientes estudiados, 21 fueron de sexo masculino (56.76\%) (Tabla 1). El 40.54\% (15) presentó antecedentes familiares de OI. De estos, 14 casos mostraron un clásico patrón de herencia autosómico dominante a través de la construcción de la genealogía (Fig. 1 A). En la mayoría de los casos estudiados, se observó solamente un individuo afectado en la familia (Fig. 1 B). La OI tipo I fue la más frecuente, con 31 casos (83.78\%) (Tabla 1). 


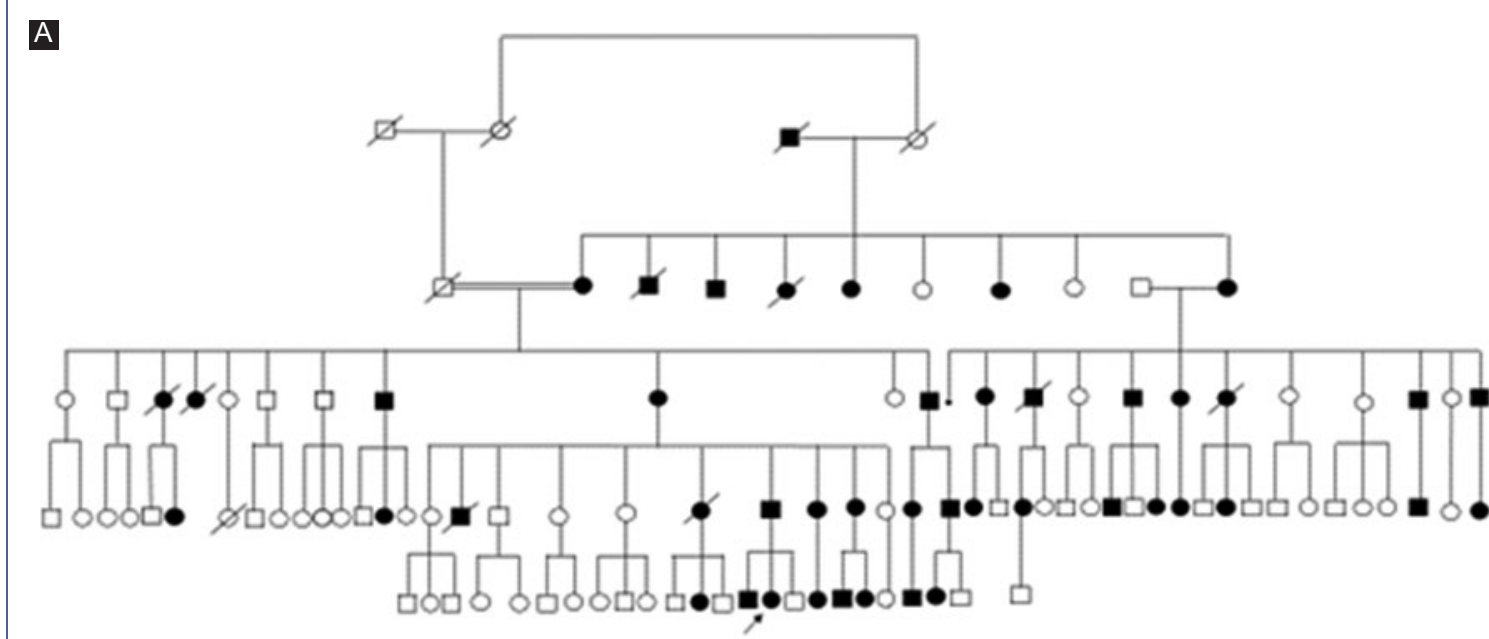

B

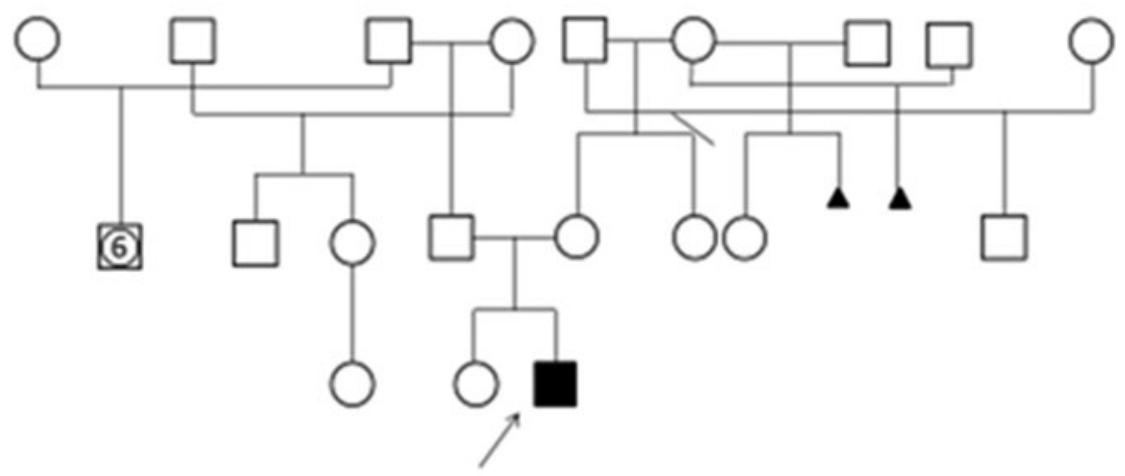

Figura 1. A: Genealogía que muestra el patrón de herencia autosómica dominante en el que se ven afectados los miembros de una familia en todas las generaciones. Se observan ambos sexos afectados por igual y la transmisión de varón a varón. La flecha señala a la paciente estudiada. B: La flecha señala el único paciente afectado en la familia.

Tabla 1. Distribución de los pacientes evaluados según el sexo y tipo de osteogénesis imperfecta

\begin{tabular}{|l|c|c|c|c|}
\hline Tipo & & Masculino & Femenino & Total \\
\hline I & IA & 11 & 9 & 20 \\
\hline & IB & 7 & 4 & 11 \\
\hline II & A & - & 2 & $2^{*}$ \\
\hline III & & 2 & 1 & 3 \\
\hline IV & IVB & 1 & - & 1 \\
\hline Total & & $21(56.76 \%)$ & $16(43.24 \%)$ & 37 \\
\hline
\end{tabular}

De forma aislada, el radio del lado derecho fue el hueso que más frecuentemente presentó fractura, y de forma conjunta, el fémur fue el más afectado, según lo referido por los representantes y registrado en la historia clínica de los pacientes (Tabla 2). Por su parte, la presencia de escleras azules fue el hallazgo que se encontró con más frecuencia, en 32 pacientes (86.49\%), seguido por la predisposición de hematomas, en 16 pacientes (43.24\%) (Tabla 3). No se pudo precisar la presencia de dentinogénesis imperfecta debido al fallecimiento precoz de dos pacientes, que correspondieron a los casos de OI tipo IIA.

El 62.16\% (23 casos) fueron referidos del Servicio de Pediatría, seguidos del $24.32 \%$ (9 casos) de los servicios de Ortopedia y Traumatología y del $13.51 \%$ (5 casos) de Endocrinología. En cuanto a su origen, 26 pacientes provenían del estado de Mérida (70.27\%) y 5 del estado de Táchira (13.51\%) (Tabla 4). Además, 31 individuos fueron procedentes del medio urbano (83.78\%). 
Tabla 2. Localización de la fractura según el hueso afectado de los pacientes evaluados con osteogénesis imperfecta

\begin{tabular}{|l|c|c|}
\hline Localización & Derecho & Izquierdo \\
\hline Clavícula & 2 & 3 \\
\hline Húmero & 5 & 5 \\
\hline Radio & 12 & 8 \\
\hline Cúbito & 10 & 8 \\
\hline Huesos de la mano & 4 & 1 \\
\hline Fémur & 10 & 11 \\
\hline Tibia & 9 & 6 \\
\hline Peroné & 4 & 3 \\
\hline Huesos del pie & 2 & 1 \\
\hline
\end{tabular}

Tabla 3. Hallazgos clínicos adicionales en los pacientes evaluados con osteogénesis imperfecta

\begin{tabular}{|l|c|c|c|}
\hline Hallazgos clínicos & Presente & Ausente & NP/NE \\
\hline Macrocefalia & 5 & 32 & - \\
\hline Escleras azules & 32 & 5 & - \\
\hline Dentinogénesis & 15 & 20 & $2^{*}$ \\
\hline Hipoacusia & 2 & 15 & 20 \\
\hline Escoliosis & 11 & 26 & - \\
\hline Hernias & 7 & 30 & - \\
\hline Deformidad & 8 & 29 & - \\
\hline Talla baja & 15 & 22 & - \\
\hline Hiperlaxitud articular & 9 & 26 & $2^{*}$ \\
\hline Hematomas & 16 & 19 & $2^{*}$ \\
\hline *Comprende los dos casos de osteogénesis imperfecta tipo IIA. & \\
\hline NP/NE: no precisado o no estudiado. & & &
\end{tabular}

\section{Discusión}

La Ol es un trastorno hereditario del tejido conectivo causado predominantemente por mutaciones heterocigotas en cualquiera de los dos genes que codifican para colágeno tipo I (COL1A1 o COL1A2) en más del $90 \%$ de los casos $^{8,9,11-13}$. No se observa diferencia en la prevalencia entre los $\operatorname{sexos}^{13}$, y los individuos presentan una amplia variabilidad fenotípica, que varía en gravedad desde formas clínicas leves a letalidad perinatal ${ }^{4,9}$. La serie de casos presentada muestra las diferentes frecuencias en los tipos de Ol, y coincide con lo documentado en la literatura, donde el tipo I es el más común. Por otra parte, la mayor presencia en el
Tabla 4. Distribución de los pacientes evaluados con osteogénesis imperfecta según el lugar de nacimiento y procedencia

\begin{tabular}{|l|c|c|}
\hline Estado & Natural & Procedencia \\
\hline Mérida & 26 & 26 \\
\hline Táchira & 5 & 6 \\
\hline Distrito Capital & 2 & 1 \\
\hline Apure & 1 & 1 \\
\hline Trujillo & 1 & 1 \\
\hline Zulia & 1 & 2 \\
\hline Exterior & 1 & - \\
\hline Total & 37 & 37 \\
\hline
\end{tabular}

sexo masculino reportada en este estudio puede deberse a que estos pacientes están más expuestos a la actividad física, lo que puede influir en el motivo de consulta.

La clasificación de Sillence divide la OI en función de la gravedad y la progresión de los síntomas: no deformantes leves (tipo I), letales perinatales (tipo II), graves progresivamente deformantes (tipo III) y moderados (tipo IV) ${ }^{8,9,14}$. Un análisis cuidadoso del patrón de herencia y el fenotipo clínico puede ayudar a guiar las pruebas genéticas cuando están disponibles ${ }^{9}$. Los avances en genética molecular han identificado genes adicionales que causan la OI, lo que ha complicado su clasificación ${ }^{8}$.

Una nomenclatura empleada para categorizar el tipo de Ol es la establecida por Bonafe, et al. ${ }^{15}$, donde la Ol se encuentra incluida dentro del grupo 25 y describe al fenotipo de forma no deformante (tipo I), perinatal letal (tipo II), progresivamente deformante (tipo III), de gravedad moderada (tipo IV) y OI con calcificación en

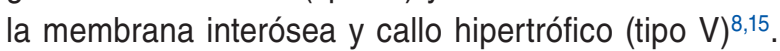
Además, se menciona que se debe utilizar una clasificación híbrida del gen causal, junto con el fenotipo clínico, para evitar la ambigüedad ${ }^{8}$. Aunque no se disponga de estudios moleculares, una evaluación clínica interdisciplinaria puede orientar el diagnóstico y la terapéutica.

La OI tipo I se origina de un defecto cuantitativo en la producción de colágeno tipo I debido a un alelo silenciado del gen $\operatorname{COL} 1 A{ }^{9}{ }^{9}$ que causa haploinsuficiencia ${ }^{8}$. Generalmente, surge como resultado de un codón de parada prematuro dentro del gen, por lo que estas mutaciones conducen a la producción de la mitad de la cantidad normal de la proteína. Por otra parte, las 
Ol tipos II, III y IV son el resultado de defectos estructurales, ocasionados por la sustitución del aminoácido glicina, que interrumpen el triple ensamblaje helicoidal del colágeno, y afectan su función e interacciones en la matriz extracelular ${ }^{8}$. Por lo tanto, surgen como defectos cualitativos y se presentan en los fenotipos más graves ${ }^{13}$.

Entre los hallazgos clínicos asociados con la Ol se encuentran la macrocefalia e hidrocefalia. Por su parte, la invaginación basilar es infrecuente, pero fatal; los síntomas incluyen cefalea, parálisis de nervios craneales inferiores, nistagmos, disfagia, cuadriparesia y ataxia ${ }^{9}$. La cifoscoliosis cervical grave ${ }^{9,16}$, aunque infrecuente, puede causar compresión de la médula espinal, cuyos síntomas incluyen trastornos sensoriales o motores de las extremidades ${ }^{8}$, así como obstrucción pulmonar y compresión de órganos ${ }^{14,17}$. La hipoacusia conductiva y neurosensorial ${ }^{9,16}$ es generalmente progresiva y se presenta en el $5 \%$ de los niños con Ol. Las escleras azules se describen particularmente en la OI de tipo I; el color puede variar, y generalmente es más oscuro en la infancia ${ }^{9}$. Por su parte, la dentinogénesis imperfecta ${ }^{9,16}$ se presenta en el $28 \%$ y se caracteriza por dientes opalescentes debido a una mayor proporción de esmalte transparente y una dentina opaca. La dentición primaria es más afectada que la permanente; las maloclusiones y la erupción dental retardada ocurren hasta en el 60$80 \%{ }^{9}$ y contribuyen al aumento del riesgo de caries ${ }^{16}$. La hiperlaxitud articular es común y puede provocar la dislocación de las articulaciones ${ }^{9,16}$ y disminuir la elasticidad de la piel, lo que facilita la aparición de hernias y un aumento de la fragilidad capilar que ocasiona hematomas $^{9}$. Los pacientes pueden presentar talla baja desproporcionada ${ }^{16}$, hipercalciuria hasta en el $36 \%$ de los casos y mayor riesgo de litiasis renal ${ }^{9}$. En adultos, la dilatación de la raíz aórtica, seguida del prolapso de la válvula mitral, es la manifestación valvular más frecuente ${ }^{9,16,17}$.

Después de la presencia de fracturas, las escleras azules y la predisposición a presentar hematomas fueron los hallazgos adicionales más frecuentes en los pacientes estudiados. Ante la pleiotropía de esta entidad, la evaluación clínica debe ser exhaustiva, incluso antes de que las complicaciones puedan evidenciarse.

Los niños que presentan lesión no accidental constituyen el diagnóstico diferencial más importante en la OI. El patrón de fracturas ocurre en costillas posteriores y metafisarias asociadas con hemorragia retiniana, que puede diferenciar la lesión no accidental de la Ol. Las fracturas de costillas se pueden encontrar hasta en el $22 \%$ de los individuos con Ol. Por otra parte, la osteoporosis juvenil idiopática también puede presentarse con historia de fracturas vertebrales frecuentes asociada con dolor óseo antes del inicio de la pubertad. Se deben considerar las causas secundarias de osteoporosis, como la malabsorción, uso de glucocorticoides, deficiencias de hormonas, leucemia linfoblástica aguda e inmovilidad, las cuales deben ser capaces de diferenciarse con base en la historia clínica y las investigaciones de laboratorio. Finalmente, otros trastornos esqueléticos que se asemejan a la OI también pueden considerarse en el diagnóstico; tal es el caso del síndrome de osteoporosis-pseudoglioma, osteoporosis idiopática juvenil ${ }^{9}$, raquitismo, hipofosfatemia ligada al cromosoma $X^{16}$, Ehlers-Danlos ${ }^{8}$, hiposfosfatasia ${ }^{9,16} \mathrm{e}$ hiperfosfatasia idiopática?

Los objetivos del tratamiento van encaminados a solucionar los problemas más frecuentes que se evidencian en la Ol, y buscan aumentar la masa ósea, disminuir el número de fracturas, evitar la talla baja y lograr la normalidad en la vida cotidiana ${ }^{18}$. Las diferentes opciones disponibles del tratamiento deben ser individualizadas en cada caso y se requiere igualmente de la evaluación de un equipo médico interdisciplinario.

La OI representa el principal motivo de consulta de alteración en el sistema esquelético en la UGM-ULA.

En esta serie de casos de OI, se resalta la amplia forma clínica de presentación de esta enfermedad, por lo que su evaluación debe ser individual e interdisciplinaria, incluso en los diferentes individuos afectados de una misma familia. Además de brindar la atención médica adecuada, se podrá brindar el oportuno asesoramiento genético familiar a través de su seguimiento y estudio.

\section{Responsabilidades éticas}

Protección de personas y animales. Los autores declaran que para esta investigación no se han realizado experimentos en seres humanos ni en animales.

Confidencialidad de los datos. Los autores declaran que han seguido los protocolos de su centro de trabajo sobre la publicación de datos de pacientes.

Derecho a la privacidad y consentimiento informado. Los autores han obtenido el consentimiento informado de los pacientes o individuos referidos en el artículo. Este documento obra en poder del autor de correspondencia.

\section{Conflicto de intereses}

Los autores declaran no tener ningún conflicto de intereses. 


\section{Financiamiento}

Ninguno.

\section{Agradecimientos}

A la licenciada Rosalía Gumina F, directora de la Biblioteca del Instituto Autónomo Hospital Universitario de Los Andes, Universidad de Los Andes, Mérida, Venezuela.

\section{Bibliografía}

1. Tsimicalis A, Boitor M, Ferland CE, Rauch F, Le May S, Carrier JI, et al. Pain and quality of life of children and adolescents with osteogenesis imperfecta over a bisphosphonate treatment cycle. Eur J Pediatr. 2018;177:891-902.

2. Leali PT, Balsano M, Maestretti G, Brusoni M, Amorese V, Ciurlia E, et al. Efficacy of teriparatide vs. neridronate in adults with osteogenesis imperfecta type I: a prospective randomized international clinical study. Clin Cases Miner Bone Metab. 2017;14:153-6.

3. Forlino A, Marini JC. Osteogenesis imperfecta. Lancet. 2016;387:1657-71.

4. Biggin A, Munns CF. Osteogenesis imperfecta: diagnosis and treatment. Curr Osteoporos Rep. 2014;12:279-88.

5. Sillence, DO, Senn A, Danks DM. Genetic heterogeneity in osteogenesis imperfecta. J Med Genet. 1979;16:101-16.

6. Van Dijk FS, Sillence DO. Osteogenesis imperfecta: clinical diagnosis, nomenclature and severity assessment. Am J Med Genet A. 2014;164A:1470-81.
7. Marginean O, Tamasanu RC, Mang N, Mozos I, Brad GF. Therapy with pamidronate in children with osteogenesis imperfecta. Drug Des Devel Ther. 2017;11:2507-15.

8. Biggin A, Munns CF. Long-term bisphosphonate therapy in osteogenesis imperfecta. Curr Osteoporos Rep. 2017;15:412-18.

9. Harrington J, Sochett E, Howard A. Update on the evaluation and treatment of osteogenesis imperfecta. Pediatr Clin North Am. 2014;61:1243-57.

10. Caparros-Martin JA, Aglan MS, Temtamy S, Otaify GA, Valencia M, Nevado J, et al. Molecular spectrum and differential diagnosis in patients referred with sporadic or autosomal recessive osteogenesis imperfecta. Mol Genet Genomic Med. 2017;5:28-39.

11. Feehan AG, Zacharin MR, Lim AS, Simm PJ. A comparative study of quality of life, functional and bone outcomes in osteogenesis imperfecta with bisphosphonate therapy initiated in childhood or adulthood. Bone. 2018;113:137-43

12. Cabral WA, Perdivara I, Weis M, Terajima M, Blissett AR, Chang W, et al. Abnormal type I collagen post-translational modification and crosslinking in a cyclophilin B KO mouse model of recessive osteogenesis imperfecta. PLoS Genet. 2014;10:e1004465

13. Tournis $S$, Dede AD. Osteogenesis imperfecta-a clinical update. Metabolism. 2018;80:27-37.

14. Escribano-Rey RJ, Duart-Clemente J, Martínez de la Llana O, Beguiristáin-Gúrpide JL. Osteogenesis imperfecta: treatment and results of a case series. Rev Esp Cir Ortop Traumatol. 2014:58:114-19.

15. Bonafe L, Cormier-Daire V, Hall C, Lachman R, Mortier G, Mundlos S et al. Nosology and classification of genetic skeletal disorders: 2015 revision. Am J Med Genet A. 2015;167A:2869-92.

16. Hoyer-Kuhn H, Netzer C, Semler O. Osteogenesis imperfecta: pathophysiology and treatment. Wien Med Wochenschr. 2015;165:278-84.

17. Marini JC, Forlino A, Bächinger HP, Bishop NJ, Byers PH, Paepe A, et al. Osteogenesis imperfecta. Nat Rev Dis Primers. 2017:3:17052.

18. Serrano R, Cammarata-Scalisi F, Briceño Y, Hernández GA. Osteogénesis imperfecta: presentación de un caso y revisión de terapias emergentes. Rev Venez Endocrinol Metab. 2018;16:53-8. 